\title{
Theoretical Analysis of Fe K-edge XANES on
}

\section{Iron Pentacarbonyl}

Wei-Ting Chen, ${ }^{a}$ Che-Wei Hsu, ${ }^{a}$ Jyh-Fu Lee, ${ }^{b}$ Chih-Wen Pao, ${ }^{b}$ I-Jui Hsu ${ }^{\star a, ~ c}$

a Department of Molecular Science and Engineering, National Taipei University of

Technology, Taipei 10608, Taiwan.

b National Synchrotron Radiation Research Center, Hsinchu 30076, Taiwan.

${ }^{c}$ Research and Development Center for Smart Textile Technology, National Taipei University of Technology, Taipei 10608, Taiwan.

*Tel.: +886-2-27712171\#2420. E-mail: ijuihsu@mail.ntut.edu.tw

KEYWORDS: TDDFT, XANES, FDMNES, FEFF, pi acceptor ligand, molecular orbital 


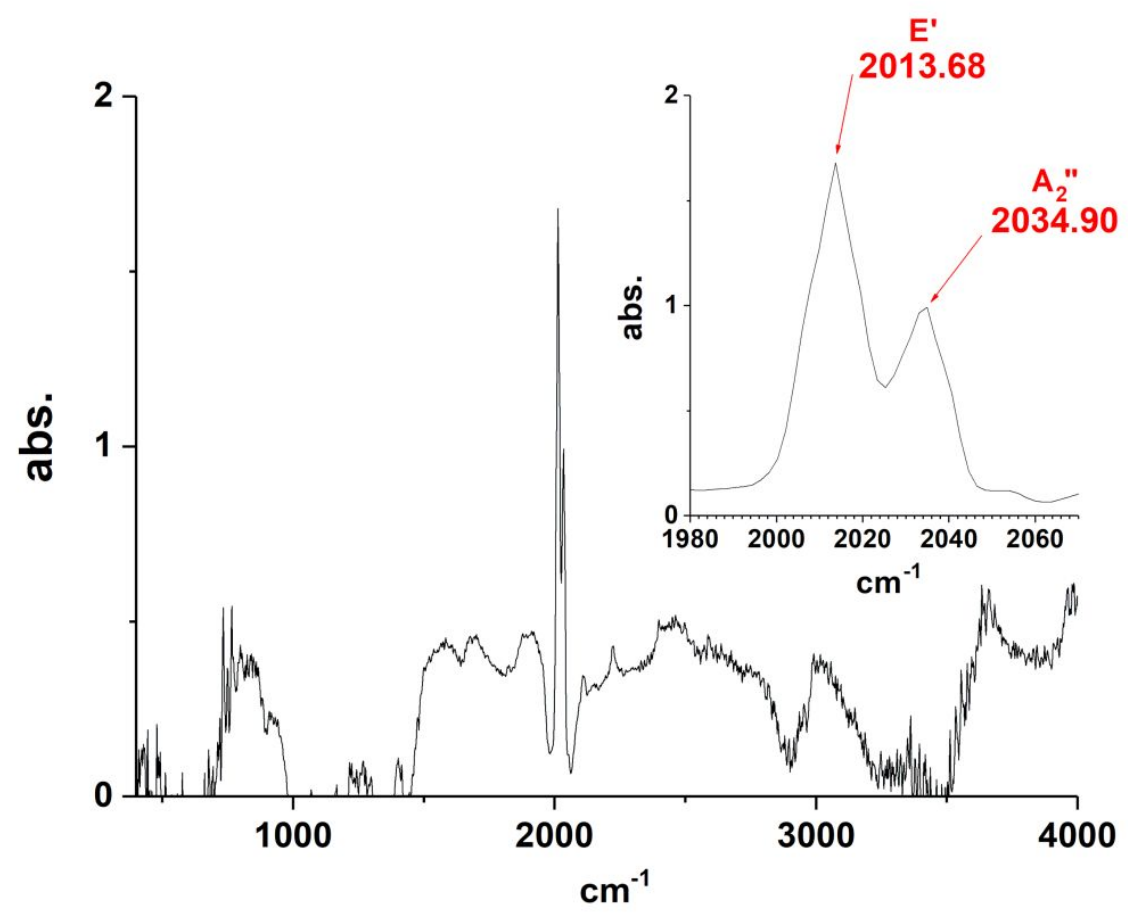

Figure S1 The IR spectra of $\mathrm{Fe}(\mathrm{CO})_{5}$ measured by reflection mode. Inset plot is an enlarged graph of $\mathrm{C} \equiv \mathrm{O}$ stretching mode near $2000 \mathrm{~cm}^{-1}$ region. We can find two infrared active modes corresponding to the $C \equiv O$ stretching at $2013.68 \mathrm{~cm}^{-1}$ and $2034.90 \mathrm{~cm}^{-1}$, respectively. 


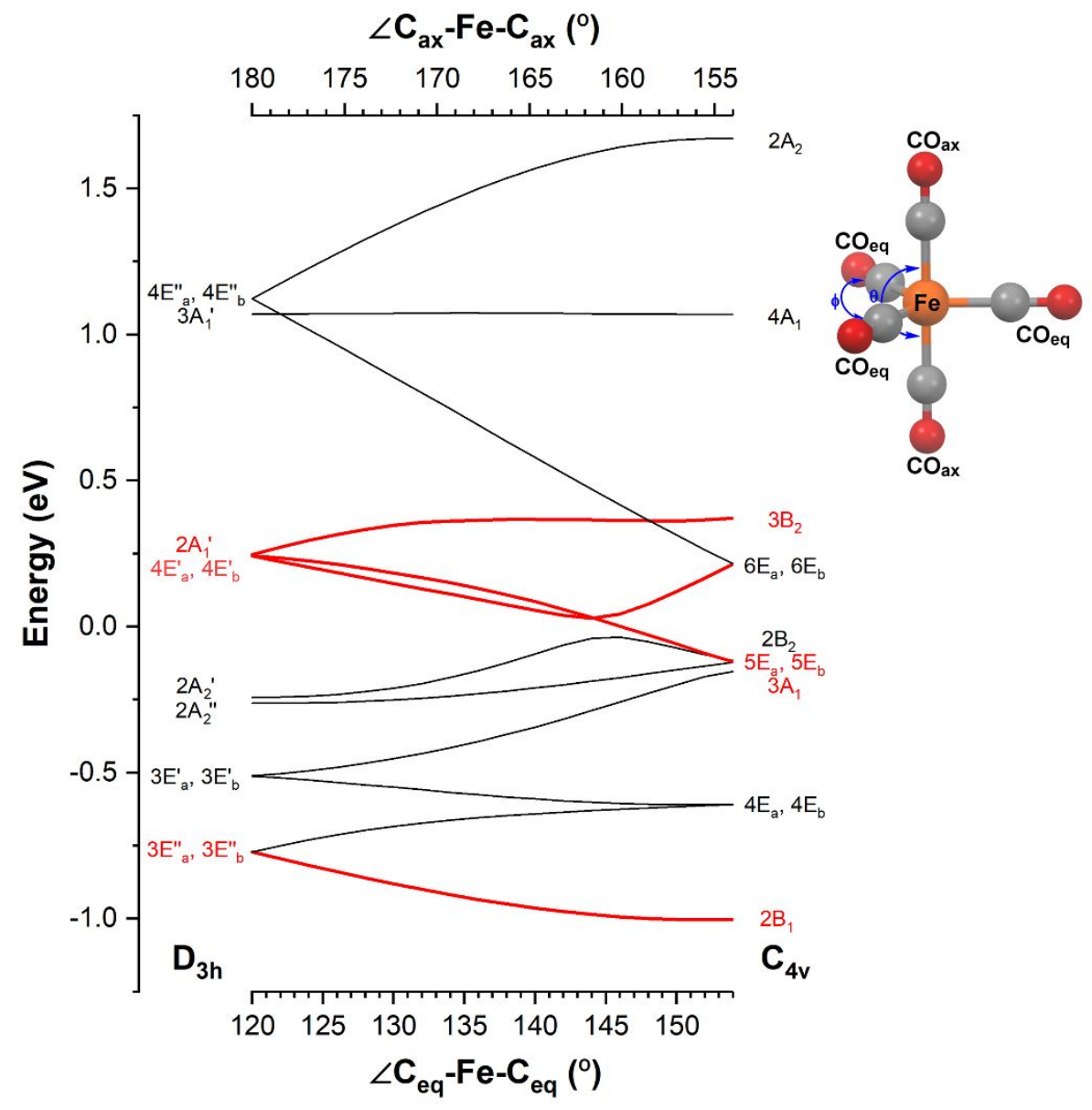

Figure S2. The variation of unoccupied MOs from $D_{3 h}$ to $C_{4 v}$. From $D_{3 h}$ structure to $C_{4 v}$ structure, each scan step decreases $\angle \mathrm{C}_{a x}-\mathrm{Fe}-\mathrm{C}_{\mathrm{ax}}$ angle by $-1.53^{\circ}$ and increases one of $\angle \mathrm{C}_{\mathrm{eq}}-\mathrm{Fe}-\mathrm{C}_{\mathrm{eq}}$ by $2.00^{\circ}$ simultaneously. 


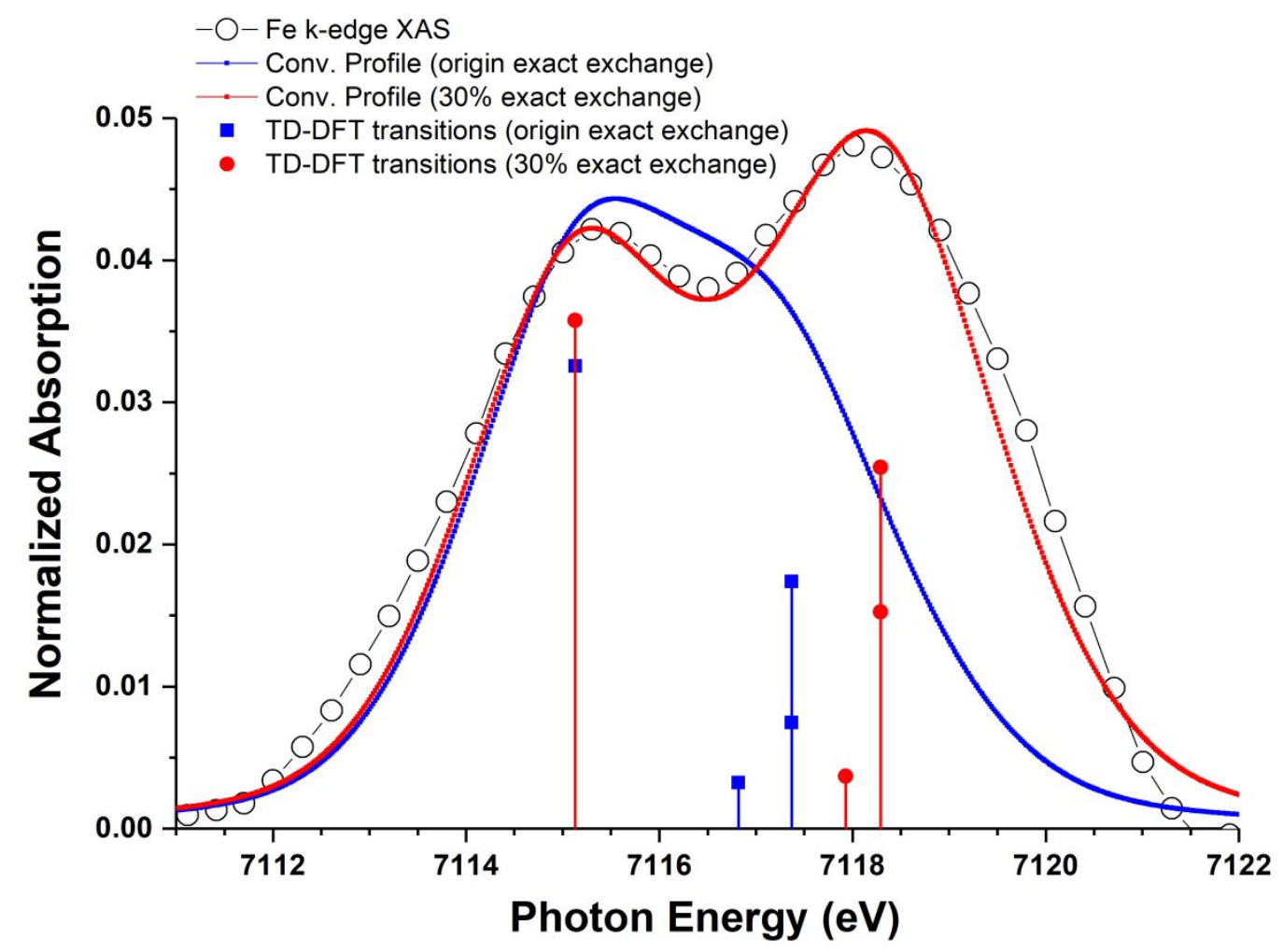

Figure S3. Comparing pre-edge of Fe K-edge XANES calculated by origin (20\%) exact exchange (blue dot and line) and 30\% exact exchange (red dot and line) of TDDFTB3LYP method. The theoretical calculated transition peaks are shown in the vertical line. All transitions have been convoluted with a pseudo-Voigt function with 1:1 ratio of Lorentzian to Gaussian and of $1.3 \mathrm{eV}$ half-width to account for experimental and core-hole broadening. The TDDFT transition energies of origin and 30\% exact exchange are shifted by $11.6 \mathrm{eV}$ and $29.55 \mathrm{eV}$. The TDDFT-B3LYP of 30\% exact exchange provides better pre-edge features. 
Table S1. The dipole and quadrupole contributions to transition intensity in $\mathrm{D}_{3 \mathrm{~h}}$ symmetry $\mathrm{Fe}(\mathrm{CO})_{5}$.

\begin{tabular}{|l|c|c|c|c|}
\hline & \multicolumn{2}{|c|}{$\begin{array}{c}\text { B3LYP-origin (20\%) exact } \\
\text { exchange }\end{array}$} & \multicolumn{2}{c|}{ B3LYP-30\% exact exchange } \\
\hline & dipole (\%) & $\begin{array}{c}\text { quadrupole } \\
(\%)\end{array}$ & dipole (\%) & $\begin{array}{c}\text { quadrupole } \\
(\%)\end{array}$ \\
\hline transitions 1 & 0.0 & 100.0 & 0.0 & 100.0 \\
\hline transitions 2 & 0.0 & 100.0 & 0.0 & 100.0 \\
\hline transitions 3 & 0.0 & 100.0 & 0.0 & 100.0 \\
\hline transitions 4 & 27.5 & 71.1 & 60.5 & 38.7 \\
\hline transitions 5 & 11.7 & 67.6 & 36.3 & 45.6 \\
\hline
\end{tabular}

Table S2. The dipole and quadrupole contributions to transition intensity in $\mathrm{C}_{4 \mathrm{v}}$ symmetry $\mathrm{Fe}(\mathrm{CO})_{5}$.

\begin{tabular}{|l|c|c|}
\hline & \multicolumn{2}{|c|}{ B3LYP-30\% exact exchange } \\
\hline & dipole (\%) & $\begin{array}{c}\text { quadrupole } \\
(\%)\end{array}$ \\
\hline transitions 1 & 0.0 & 100.0 \\
\hline transitions 2 & 100.0 & 0.0 \\
\hline transitions 3 & 0.0 & 100.0 \\
\hline transitions 4 & 42.9 & 41.1 \\
\hline transitions 5 & 42.9 & 41.1 \\
\hline
\end{tabular}

